\title{
Erhöht eine Sectio caesarea das Allergierisiko?
}

\author{
Studien der letzten Jahre zu Folgen des Geburtsmodus \\ haben eine mikrobiologische Überraschung ergeben: \\ Kaiserschnitt-Kinder weisen eine andere intestinale \\ Mikroflora auf als auf natürlichem Wege geborene Kin- \\ der. Auch die Darmflora allergischer Kinder unterscheidet \\ sich von Gesunden. Die Frage lautet also: Könnte eine \\ Sektio auch das Risiko für allergische Rhinitis und \\ Asthma beeinflussen?
}

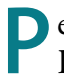
er Telefoninterview wurden 9.722 Däninnen aus einer laufenden Kohortenstudie zwischen 1997 und 2001 zu Allergien und Asthma befragt. Für die Probandinnen lagen detaillierte Informationen zu Art der Geburt, Gestationsalter, Alter der Mutter und Anzahl vorausgegangener Schwangerschaften vor.

94,9\% der Frauen waren auf natürlichem Wege, 5,1\% durch Kaiserschnitt zur Welt gekommen. Von den Frauen litten $14,0 \%$ (1.272) an Heuschnupfen und $11,4 \%$ (1.038) an Asthma bronchiale. Für allergische Rhinitis konnten die Autoren keinen $\mathrm{Zu}$ sammenhang zwischen erhöhtem Aller- gierisiko und Geburtsweg oder sonstigen Geburtsfaktoren feststellen. Lediglich die Anzahl vorausgegangener Geburten hatte einen negativen Einfluss $(\mathrm{p}=0,0001)$ auf die Entwicklung von allergischer Rhinokonjunktivitis (bekanntermaßen verringert sich die Allergiehäufigkeit mit zunehmender Geschwisterzahl). Für Asthma ergab sich dagegen eine leichte, aber signifikant positive Korrelation: Frauen, die per sectionem geboren worden waren, hatten im Vergleich zu vaginal Geborenen ein 1,33-fach erhöhtes Risiko für späteres Asthma (95\%-Konfidenzintervall 1,02-1,74).

\section{Die Mutter als Allergenquelle}

\section{Der Kontakt mit der Außenwelt ist offenbar schon in utero aus- schlaggebend für die Entwicklung von Allergien. Denn die meisten Neugeborenen zeigen bereits eine T-Zellantwort gegen Nahrungs- mittel- und Aeroallergene. Die nahe liegende Hypothese, dass Aller- gene über die Plazenta zum Kind gelangen, prüften österreichische Kinderärzte in vitro.}

\footnotetext{
Dädiater von der Universität Wien verwendeten frische, intakte $\mathrm{Pla}$ zenten von Kaiserschnitt-Kindern und prüften die Übertragung von $\beta$-Laktoglobulin aus Kuhmilch, des Hühnereiweißes Ovalbumin sowie des Birkenpollenallergens Bet v1 aus dem mütterlichen in den fetalen Blutkreislauf. Dazu wurden die gelösten Allergene in
}

die maternalen Spiralarterien der Plazenta injiziert und das Perfusat im venösen Abfluss der fetalen Plazenta auf übertragene Allergene untersucht. Die Intaktheit des plazentalen Kreislaufs wurde durch Perfusion mit einem nicht passagierenden Farbstoff kontrolliert.

Alle drei Allergene fanden sich in zunehmenden Mengen im fetalen Per- fusat. Die Konzentrationen der maternalen Allergenlösungen lagen im $\mathrm{mg} / \mathrm{ml}$ Bereich, auf der fetalen Seite wurden Werte zwischen 1 und 150 $\mathrm{ng} / \mathrm{ml}$ erreicht.

Fazit: Allergene können offensichtlich aus dem mütterlichen Blutkreislauf über die Plazenta zum Feten gelangen. Diese Planzentagängigkeit könnte die bereits pränatal vorhandene Immunreaktion auf bestimmte Allergene erklären. $\mathrm{Ob}$ sich auf diese Art eine allergische Disposition etablieren kann, ist allerdings offen. $b k$

Loibichler C et al. Materno-fetal passage of nutritive and inhalant allergens across placentas of term and pre-term deliveries perfused in vitro. Clin Exp Allergy 2002; 32: 1546-51 